\section{NEW FLUORESCENCE METHODOLOGY FOR DETECTING DNA ADDUCTS}

Progress Report

Project Period: 5/1/91 - 11/30/93

Period Covered by this Report: 5/1/91 - 5/21/93

Roger W. Giese

Northeastern University

Boston, Massachusetts 02115

May 21, 1993

Prepared for

THE U.S. DEPARTMENT OF ENERGY

AGREEMENT NO. DE-FG02-9OER60964

\section{RECEIVED JUN 141993 OSTI}
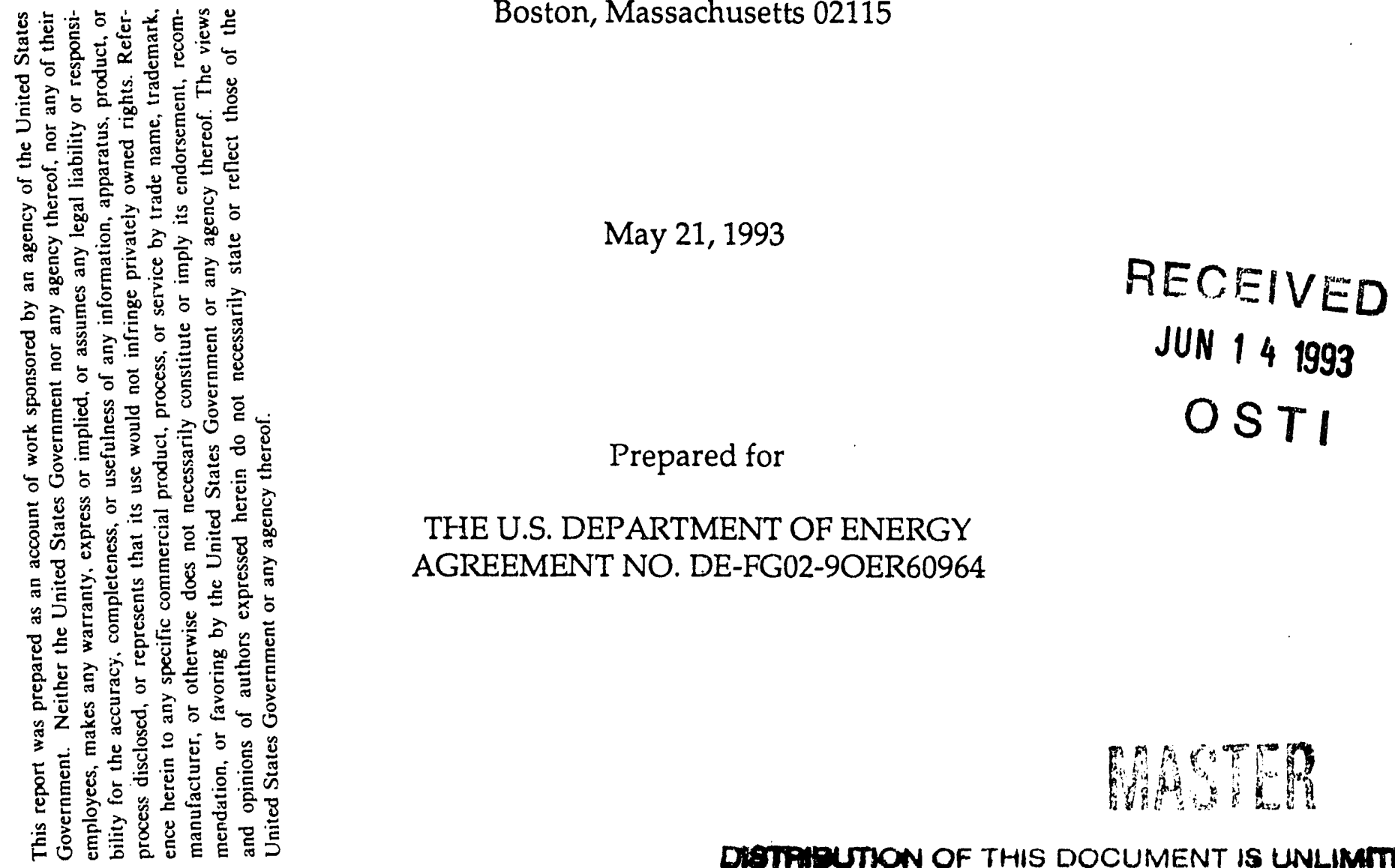


\section{DOE Progress Report}

\section{$\underline{\text { Abstract }}$}

We believe that our start-up project has been successful. Most importantly, a new reagent, "BO-IMI", has been developed that achieves, for the first time, single step, phosphate specific fluorescence labeling under aqueous conditions. Both $3^{\prime}$ and $5^{\prime}$ mononucleotides, including representative DNA adducts can be labeled. Included in this technique is a convenient procedure for postlabeling sample cleanup, leading to a practical detection of the products by capillary electrophoresis with laser fluorescence detection (CE-LIF). We consider that this new method will have a significant impact on the measurement of DNA adducts in human samples. This work was largely accomplished in the second half of our project. In the first half, we set up a new way to isolate DNA nucleotides from blood, worked with an initial, less specific technique for labeling DNA adducts, compared ionizing radiation vs oxidative damage to fluorescein labeled deoxyadenylic acid, and set up a capillary electrophoresis laser fluorescence detection system.

\section{Background}

In this project, we are developing new methodology to detect DNA adducts. The purpose of the methodology is to improve our ability to investigate chemical damage to human DNA. This requires that the measurements are achieved with high sensitivity, due to the limited amounts of DNA in hiıman samples DNA may be genotoxic.

The proposed new methodology comprises the following sequence of analytical steps: (1) purify the DNA from a biological sample; (2) convert the DNA to deoxynucleotides; (3) isolate the deoxynucleotide DNA adducts from the bulk of the normal deoxynucleotides; (4) covalently label the DNA adducts with a fluorophore; and (5) detect the adducts by capillary electrophoresis with laser fluorescence detection.

In concept, the new methodology is the same as ${ }^{32} \mathrm{P}$ post-labeling TLC, a successful method for the detection of DNA adducts. Nevertheless, the new methodology is intended to overcome some serious shortcomings of the ${ }^{32} \mathrm{P}$ method.

First of all, the $32 \mathrm{P}$ method, because of the radioactivity, is not convenient for detecting polar adducts. It's real strength is bulky, nonpolar adducts. Thus the $32 \mathrm{P}$ method only reveals some of the adducts which are present, a major shortcoming. Our new method, since it avoids radioisotopes, putentially can be made convenient for the detection of all kinds of adducts. 
Second, ${ }^{32} \mathrm{P}$ relies on enzymatic labeling of the adducts. Unfortunately, adducts tend to differ in their susceptibility to this type of labeling. For example, the major cisplatin-DNA adduct has been reported to undergo labeling with $32 \mathrm{P}$ only $0.1 \%$ as efficiently as the corresponding dinucleotide (Hemminki, K., Peltonin, K. and Mustonin, R., 1990, Chem.-Biol. Interact. 74, 45-54). This is a second major shortcoming that our method promises to overcome. We instead rely on a chemical reaction, instead of an enzymatic one, for attaching our label (a fluorescent dye) to the DNA adducts.

Third, TLC-autoradiography is the only practical detection technique for ${ }^{32} \mathrm{P}$ post-labeling, and this is how the ${ }^{32} \mathrm{P}$ method is practiced in nearly all laboratories which utilize this method. But this is a low resolution technique. Multiple DNA adducts are seen as a pile of superimposed black spots on the diagonal of the TLC plate. Our new method will take advantage of the high resolution of capillary electrophoresis.

Finally, it is attractive that we will eliminate the handling, cost and disposal problems of radioisotopes by using instead a fluorescent tag.

\section{BO-IMI (Manuscripts 1 and 2).}

As presented in more detail in Appendix A (Manuscripts 1 and 2) BO-IMI is an exciting new reagent for the detection of DNA adducts by capillary electrophoresis with laser fluorescence detection. A patent application has been filed by Northeastern University for this invention, which will be licensed and marketed by Molecular Probes. The reagent achieves single step, phosphate specific labeling under mild aqueous conditions. The labeling reaction is easily coupled to capillary electrophoresis (CE). The ability of $C E$ to resolve some model and representative DNA adducts has been demonstrated. A trace enrichment injection technique has been set up for $C E$ that, even at a preliminary stage, reaches a low attomole level of detection for a realistic sample (injection volume $3.5 \mathrm{ul}$ ). Specificity of the labeling reaction has been demonstrated: neither glycine or albumin, when added to the labeling reaction, interfere with the yield, nor give rise to additional fluorescence labeled products. It is clear that the performance that BO-IMI achieves for fluorescence labeling DNA adducts particularly the specificity and convenience in conjunction with CE-LIF, will lead to this technology becoming significant for the measurement of DNA adducts in human samples.

DNA Nucleotides from Leukocytes: Publication 3.

We have developed a novel procedure for purifying DNA from a biological sample and converting it to deoxynucleotides. The starting biological sample to which the method was applied is leukocytes (as would be obtained from the buffy coat of blood). 
It is significant to isolate DNA adducts by a method other than a conventional procedure involving a phenolic extraction. In the latter technique artifactual DNA adducts (especially oxidative adducts) are likely to be generated during the procedure from reactive, degradation products derived from phenol, an unstable chemical. Thus the phenolic extraction is particularly unsuitable for measuring DNA adducts caused by ionizing radiation.

\section{Laser Fluorescence Detector for CE: Publication 4.}

Funded by a subcontract of our proposal, Dr. Edward Yeung at Iowa State University built a laser (argon-ion) fluorecence detector for CE. At the outset of our project, such a detector was not available commercially. This detector has been successful for our project, and in fact was selected as one of the top 100 new instruments in 1991 by IRD magazine. The initial version of this detector was susceptible to mechnical vibration that a subsequent version overcame.

\section{CE-LIF of Fluorescein-ED-Deoxynucleotides: Publication 5.}

We demonstrated that CE-LIF was powerful for the separation of fluorescence-labeled deoxynucleotides, expecially at an elevated $\mathrm{pH}$.

\section{CE Column Washing: Publication 6.}

We introduced a more convenient procedure for washing a CE column and published this as a Note.

Ionizing Radiation vs Oxidative Damage to a Fluorescein-Labeled Nucleotide: Publication 7.

We introduced a new way to search for a DNA adduct which is specific for ionizing radiation damage to DNA. The concept is to subject a fluorophorelabeled deoxynucleotide to ionizing radiation vs oxidative conditions, then compare the profiles of the product mixtures by capillary electrophoresis. In fact, a peak was observed from the ionizing radiation exposure that was not present in the sample subjected to oxidative damage. This technique deserves further attention, particularly with BO-IMI deoxynucleotide conjugates, where it could be helpful to take advantage of the reversibility of the labeling (attach fresh, undamaged BO-IMI to the irradiated deoxynucleotide products) to rule out damage to the nonnucleotide part of the conjugate. This work also confirmed our expectation that $\mathrm{CE}$ would offer high resolution for the detection of a complex mixture of fluorescent labeled deoxynucleotides. 
Synthesis of an Oxidative DNA Adduct: Publication 8.

In regard to the above study of ionizing radiation, we encountered difficulty in obtaining an authentic sample of 8-oxo- $\underline{A}$ as a deoxynucleotide. This led to our developing an improved synthesis for this adduct.

\section{Publications}

As indicated by the above, a combined total of eight manuscripts and publications has resulted to date from this project. Of the two manuscripts, one is submitted for publication and the other manuscript will be submitted shortly.

1. Wang, P. and Giese, R.W., Phosphate-Specific Fluorescent Labeling with "BO-IMI", submitted for publication.

2. Wang, P. and Giese, R.W., Capillary Electrophoresis of BO-IMI Labeled Deoxynucleotides, in preparation.

3. Al-Deen, A.N., Cecchini, D.J. and Giese, R.W., Purification of DNADerived Deoxynucleotides From Leukocytes Involving Nuclease Elution of a Ion-Exchange Column, I. Chromatogr., 600, 229-233 (1992).

4. Yeung, E.S., Wang, P., Li, W. and Giese, R.W., Laser Fluorescence Detector for Capillary Electrophoresis, I. Chromatogr., 60ㅇ, 73-77 (1992).

5. Li, W., Moussa, A. and Giese, R.W., Capillary Electrophoresis of Fluorescein-Ethylenediamine-5'-Deoxynucelotides, I. Chromatogr., 608 , 171-174 (1992).

6. Abdel-Baky, S. and Giese, R.W., Capillary Electrophoresis Washing Technique, I. Chromatogr., 608, 159 (1992).

7. Li, W., Moussa, A. and Giese, R.W., Capillary Electrophoresis with Laser Fluorescence Detection for Profiling Damage to Fluorescein-Labeled Deoxyadenylic Acid by Background, Ionizing Radiation and Hydrogen Peroxide, I. Chromatogr., 633, 315-319 (1993).

8. Abdel-Baky, S. and Giese, R.W., Improved Synthesis of 8-Hydroxy-2'Deoxyadenosine-5'-Monophosphate, Syn. Comm., 23, 861-865 (1993).

freprints and reprints - kemoved 

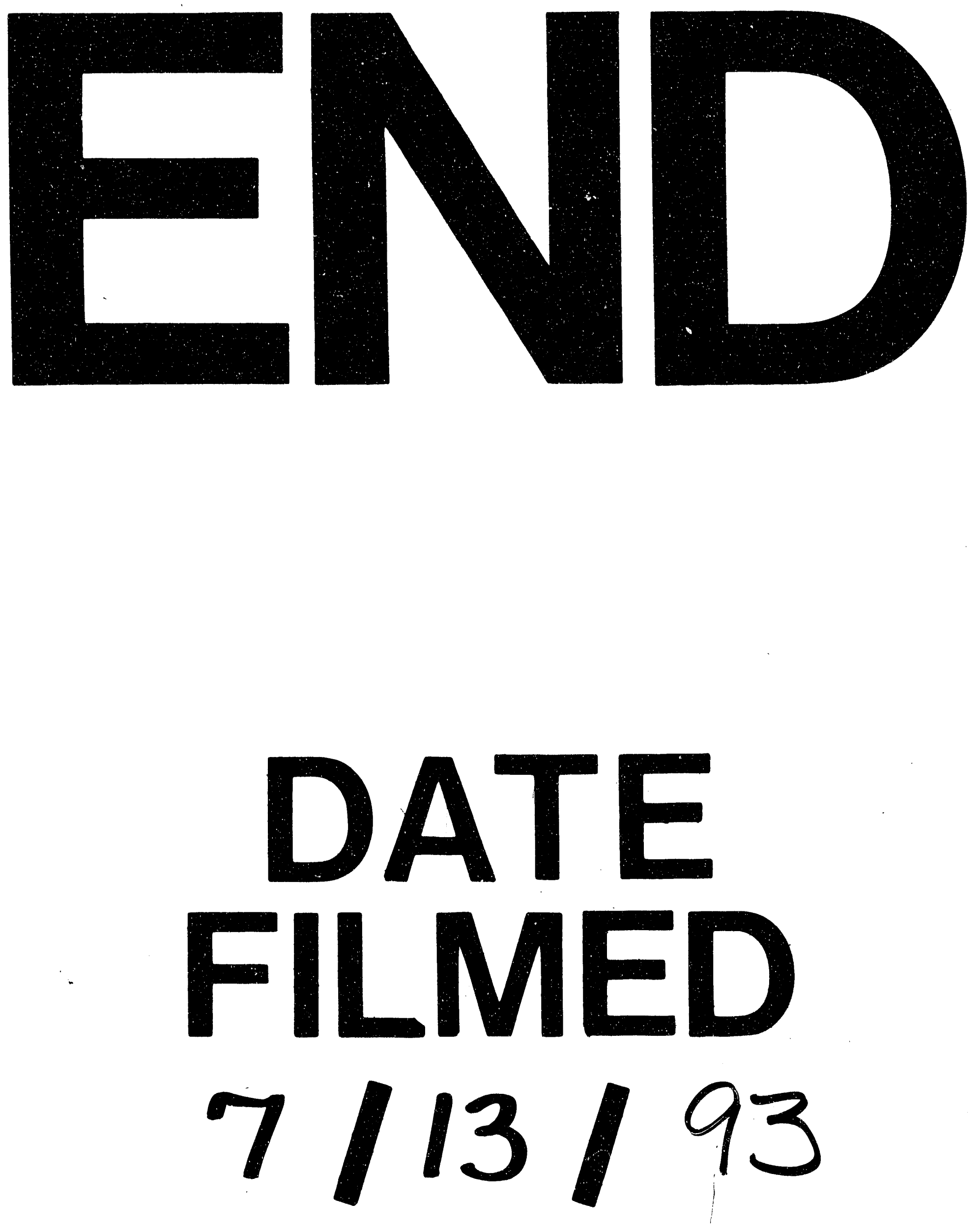
